Volume 2, Issue 1, May - 2021: pp: 33-38 www.ejor.sohag-univ.edu.eg

Doi: 10.21608/ejor.2021.175572

Original article

\title{
EVALUATING THE RESULTS OF TREATMENT OF CASES OF FRACTURE SHAFT TIBIA BY USING INTERLOCKING INTRAMEDULLARY NAIL
}

\author{
Mohamed Abdel-Wanis, Abd Elrahman Khalifa, Ahmed Saleh, Amr Abd El Gawaad ${ }^{(*)}$ \\ Orthopaedic dept, Faculty of Medicine, Sohag University, Sohag, Egypt \\ *E-mail: amr_elsamangy3000@yahoo.com
}

Received 19/1/2021

Accepted 28/4/2021

\begin{abstract}
The objective of this prospective study showing the result of fixation of fracture shaft tibia in adults by intramedullary interlocking nail tibia. Thirty patients with fracture shaft tibia simple \& compound fracture type $1 \& 2$ Gustilo-Anderson Classification. Surgical protocol of fixation of fracture shaft tibia in adults by intramedullary interlocking nail tibia Regarding union, alignment $\&$ functional results using Olerud functional scoring system. According to Olerud functional scoring system 17 patients had excellent overall functional results, 10 had good results, 2 had fair results only one patient had poor result on account of her old age and poor health condition. Intramedullary nailing is the best option. Solid core nails are associated with the lowest rate of infection. Intramedullary nailing is the current most popular form of surgical treatment. It has many advantages include: Indicated in cases of segmental and comminuted fractures shaft tibia, saving fracture hematoma, early weight baring, minimal incision, less blood loss.
\end{abstract}

Keyword: Fracture, Tibia, Interlocking, Healing, Union

\section{Introduction}

The tibia is the most commonly fractured long bone. Tibia is a subcutaneous bone, Most of these fractures are found in young males. It represents $0.2 \%$ of all injuries. Motor vehicle accidents, skiing accidents, high-energy falls and sports are the commonest causes [1-11]. Many options for treatment, conservative treatment, surgical treatment include: Plating, Interlocking nail, External fixation [1217]. The goal of this study is a prospective follow up and assessment of results of internal fixation of tibial shaft fractures by interlocking intramedullary nails reg- arding, union, alignment and the functional outcomes. Intramedullary nailing is the best option. Solid core nails are associated with the lowest rate of infection. Intramedullary nailing is the current most popular form of surgical treatment. It has many advantages include: Indicated in transverse and short oblique shaft fracture it also suitable for treatment of delayed union and pseudo-arthros is also in cases of segmental and comminuted fractures shaft tibia, saving fracture hematoma, early weight baring, minimal incision, less blood loss [18-24]. 


\section{Patents and Methods}

It is a prospective study of 30 adult patients with tibial shaft fractures admitted in Orthopedics and Traumatology dep. Sohag Univ. hospital, from July 2014 till March 2015. Criteria of included participants: Skeletally mature patients. Simple racture shaft tibia and Gustilo-Anderson Classification type $1 \& 2$. AO classification type A \& B \& C. surgical fit patients. Exclusion criteria: Skeletally immature patients. Gustilo-Anderson Classification type 3. Surgical unfit patients. In all the patients along with personal data, mode of trauma, type of fracture, type of surgery, intra operative \& post-operative complications, follow up examination, timing of full weight bearing were considered.

\subsection{Management of patients}

As soon as the patient with suspected tibial shaft fracture was seen, necessary clinical and radiological evaluation done and admitted to the ward after necessary resuscitation and splint age using above knee slab. The following investigations were done routinely on all the patients preoperatively: Complete blood count and differential count. Prothrombin time and concentration. Blood grouping and cross matching. Blood glucose level. Blood urea and serum creatinine.

\subsection{Radiological}

$\mathrm{X}$-rays: Leg with both knees AP view and lateral view, fig. (1)

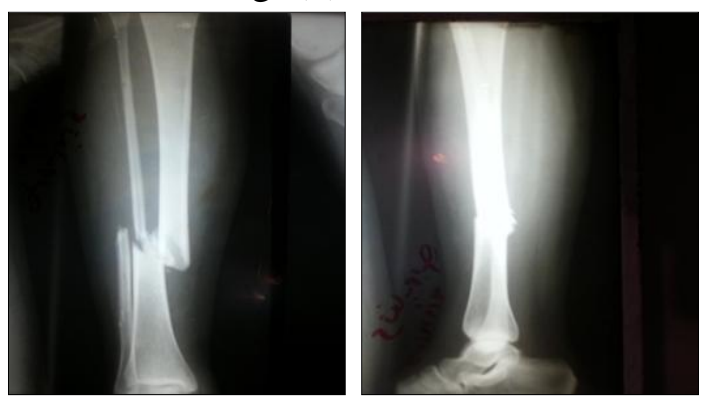

Figure (1) shows Preoperative X-ray: A25-yearsold male with fracture both bone rt leg AP\&LAT views.
The patient was placed in supine position on fracture radiolucent table and was ope1rated upon spinal anesthesia. Para patellar or splitting of patellar tendon was done, fig. 2), reaming was done in all cases, fig. $(3,4)$. Length was measured by placing radio opaque ruler.

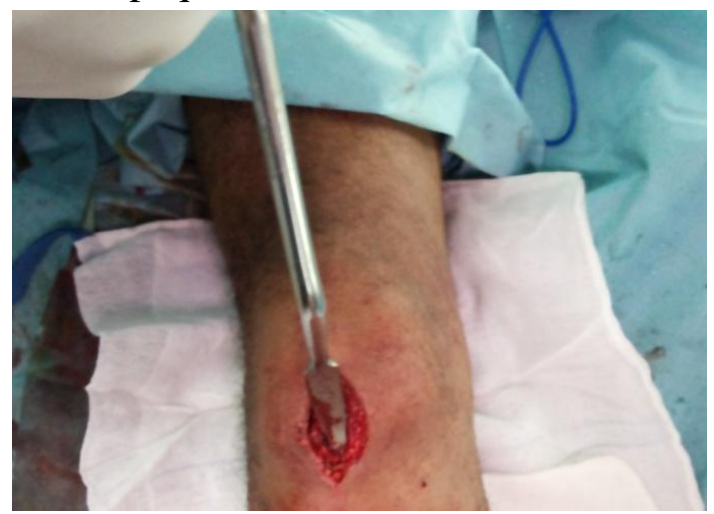

Figure (2) shows tibial opening by awl

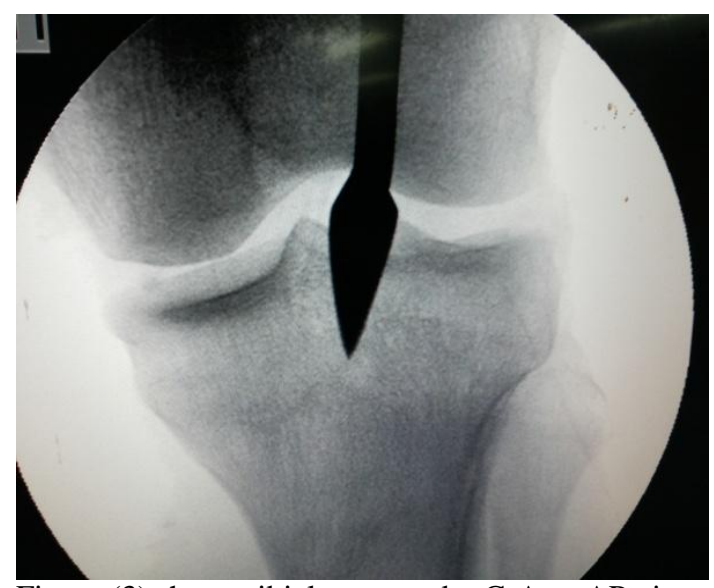

Figure (3) shows tibial entry under C-Arm AP view
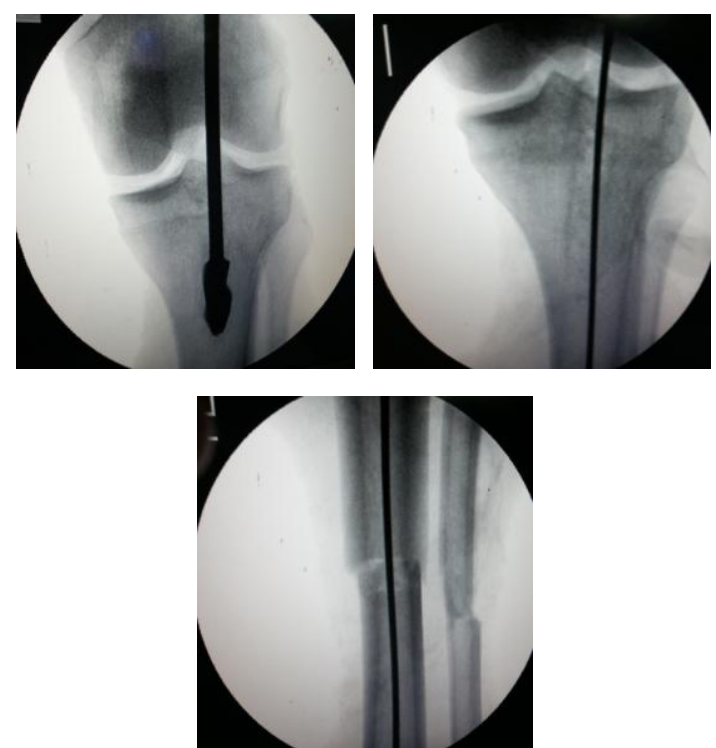

Figure (4) shows entry with the guide wire 
Postoperatively foot and leg elevation is allowed. Antibiotics were continued in the post-operative period, I.V for 3 days and oral antibiotics for 1 week. Analgesics were given when indicated. Sutures removed on $14^{\text {th }}$ postoperative day. After operation, patients were partially weight-bearing (15 to $20 \mathrm{~kg}$ ) for six to eight weeks. Thereafter, weight-bearing was increased based on the absence of pain and a study of the radiographs on follow-up, fig. (5). Early active knee and ankle range-ofmotion exercises were encouraged in all patients who were compliant. Patients were discharged from the hospital 4-5 days postoperatively. All patients were followed up at 2 weeks to remove stitches. 1 month, 3 months, 6 months, 9 months.

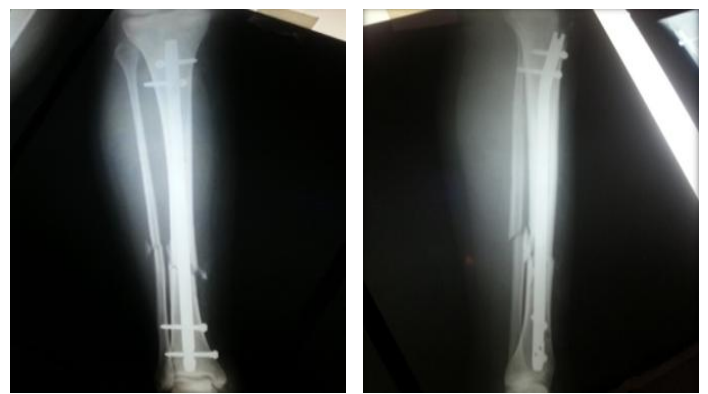

Figure (5) shows postoperative X-ray AP \& LAT views.

\subsection{Clinical evaluation}

The clinical examination included physical examination, including a neurovascular examination and assessment of axial alignment, and a functional analysis. Functional recovery was assessed using OlerudMolanderankel score. Olerud-Molanderankel score of 91 to 100 points was considered excellent; $61-90$ points, good; 31-60 points, fair; and less than 31, poor. At every visit patients were examined using x-ray to asses fracture union and clinically regarding knee and ankle function, walking ability, deformity and limb length discrepancy.

\section{Results}

There were 27 male and 3 female (ratio 9:1) with mean age 34 (20:60). Motor car accident represents $75 \%$ ( 21 cases) of mode of trauma. 21 patients have simple fractures $(70 \%)$ and 9 patients have open fractures (30\%), 5 patients grade 1 and 4 patients grade $2.70 \%$ were operated within 24 hours following admission in hospital. But in some patients operative procedure was delayed due to medical problems (Hypertension, diabetes and ischemic heart disease) and financial constraints of patients. Six patients have associated head injuries and eight patients have other fractures at humerus, radius, and femur. Also internal fixation is done for these fractures. Average time for which patient was admitted in Sohag university hospital was 5 days postoperatively. Patients were discharged 2-3 days post-operative. Duration of surgery was more for the initially operated cases. Mean time in $(\mathrm{min})$ for cases operated by interlocking nail was $65 \mathrm{~min}$. According to Olerud functional scoring system 17 (57\%) patients had excellent overall functional results, 10 (33\%) had good results, 2 (7\%) had fair results only one (3\%) patient had poor result on account of her old age and poor health condition. One of the thirty patients had postoperative varus fracture angulation that was less than 5 degrees in the coronal and sagittal planes and one patient had postoperative valgus angulation of about $5^{\circ}$, fig. (6).

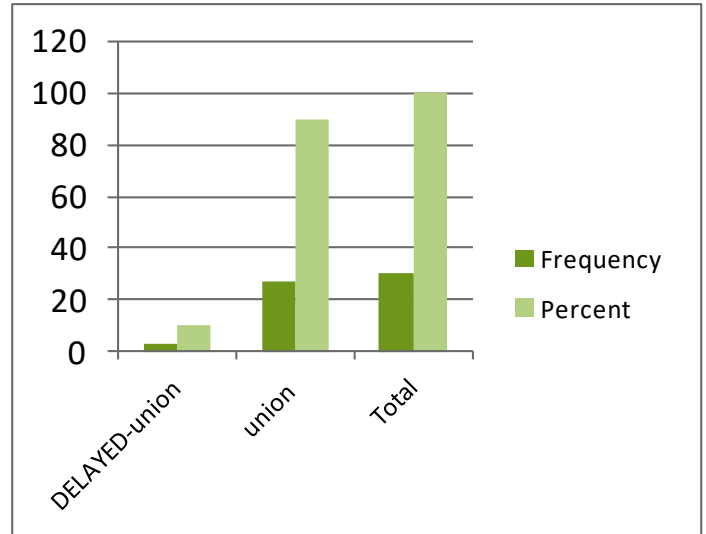

Figure (6) shows chart represents post-operative Union 
Average time of union in all our 30 patients was about 13 weeks (Range: 10 to 26 weeks) $(\mathrm{SD}=5)$. Three cases had delayed union (10\%), fig. (7), dinamization was done and union appeared after 2 months. The three cases were open fractures type 2. Superficial would infection was seen in two cases. The infection noticed one week later and IV antibiotics was given and this superficial would infection completely cured. Two recorded cases of deep wound infection and wound hematoma in two weeks postoperatively, debridment done and IV antibiotics was given and patients completely cured.

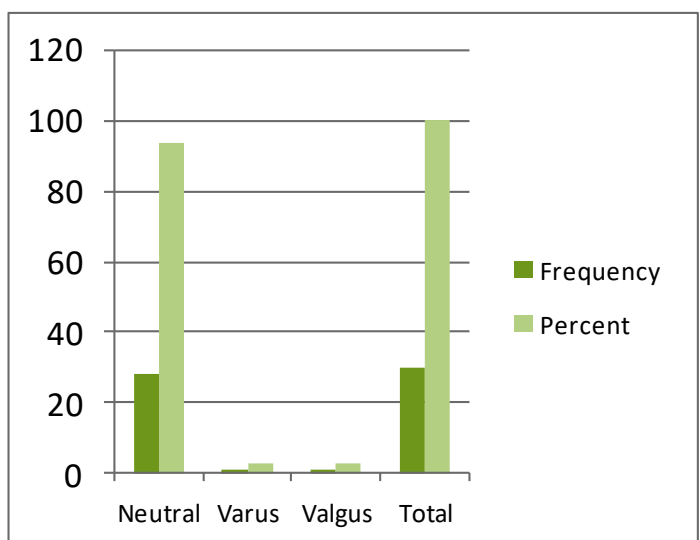

Figure (7) shows chart represents rotational malalignment

\section{Discussion}

Tibial shaft fractures are more commonly seen in young people particularly in $2^{\text {nd }}$ to $4^{\text {th }}$ decades of life. Common mechanism of injury is seen following high energy trauma like Road traffic accident (RTA), fall from height etc. In older patients apart from osteoporosis as major risk factor, anemia, cachexia, cognitive dysfunction, visual impairment, social dependence are some other factors which makes these people more prone for such fractures [13]. In this study an attempt was made to evaluate the management of those individuals by using Interlocking tibial nail (IMN). There is nine cases have open fractures, six of them type 1 and the rest is type 2 . Also there is 21 cases have simple fractures. None of the thirty cases in our study was subjected to reoperation during their follow up. No recorded cases of nonunion.

\section{Conclusion}

Finally we conclude that closed intramedullary interlocked nailing for tibial fractures gives excellent results in closed and grade $1 \& 2$ open fractures if done judiciously and with proper technique. Moreover it offers significant benefits to the patient in the form of short hospital stay, early range of motion exercises, also partial weight bearing can be started early, so both function and healing of fracture goes side by side and thereby, consequent good functional results were achieved in a majority of these fractures

\section{References}

[1] Schmidt, A., Finkemeier, C., Tornetta $3^{\text {rd }}$, P. (2003). Treatment of closed and open tibial fractures. Instr Course Lect. 52: 607- 622

[2] Gustilo, R., Anderson, J. (1976). Prevention of infection in the treatment of one thousand and twenty-five open fractures of long bones: Retrospective and prospective analyses. J. Bone Joint Surge Am. 58 (4): 453-458.

[3] Brumback, R., Jones, A. (1994). Interobserver agreement in the classification of open fractures of the tibia. The results of a survey of two hundred and fortyfive orthopaedic surgeons. J. Bone Joint Surg Am. 76 (8): 1162-1166.

[4] Gustilo, R., Gruninger, R., Davis, T. (1987). Classification of type III (severe) open fractures relative to treatment and results. Orthopedics. (12): 17811788.

[5] Tripuraneni, K., Ganga, S., Quinn, R., et al. (2008). The effect of time delay to surgical debridement of open tibia 
shaft fractures on infection rate. Orthopedics. 31 (12), R ID: 32925.

[6] Kindsfater, K., Jonassen. E. (1995). Osteomyelitis in grade II and III open tibia fractures with late debridement.

J. Orthop Trauma. 9 (2):121-127.

[7] Tornetta, P. (1997). Tibial fractures. In: Dee, R., Hurst, L., Gruber, M., et al. (eds.) Principles of Orthopedic Practice, McGraw-Hill, NY, pp. 519-530.

[8] Lefaivre, K., Guy, P., Chan, H., et al. (2008). Long-term follow-up of tibial shaft fractures treated with intramedullary nailing. J. Orthop Trauma. 22 (8): 525-529.

[9] Agrawal, A., Chauhan, V., Maheshwari. R., et al. (2013). Primary nailing in the open fractures of the tibia-is it worth? J. Clin Diagn Res. 7 (6): 1125-1130.

[10] Riemer, B., Di Christina, D., Cooper, A., et al. (1995) Nonreamed nailing of tibial diaphyseal fractures in blunt polytrauma patients. J. Orthop Trauma. 9 (1): 66-75.

[11] Vallier, H., Le, T., Bedi, A. (2008). Radiographic and clinical comparisons of distal tibia shaft fractures (4-11 $\mathrm{cm}$ proximal to the plafond): Plating versus intramedullary nailing. $\boldsymbol{J}$. Orthop Trauma. 22: 307- 311.

[12] Burc, H., Dursun, M., Orhun, H., et al. (2009). Treatment of adult tibial diaphysis fractures with reamed and locked intramedullary nailing. Acta Orthop Traumatol Turc. 43: 7-13.

[13] Krettek, C., Rudolf, J., Schandelmaier, P., et al. (1996). Unreamed intramedullary nailing of femoral shaft fractures: Operative technique and early clinical experience with the standard locking option. Injury. 27: 233-254.

[14] Liporace, F., Stadler, C., Yoon, R. (2012). Problems, tricks, and pearls in intramedullary nailing of proximal third tibial fractures. J. Orthop Trauma. 27

(1): 56-62

[15] Krettek, C., Miclau, T., Schandelmaier, P., et al. (1999). The mechanical effect of blocking screws ("Poller screws") in stabilizing tibia fractures with short proximal or distal fragments after insertion of small diameter intramedullary nails. J. Orthop Trauma. 13: 13 (8): 550-553

[16] Hicks, C., Noble, P., Tullos, H. (1995). The anatomy of the tibial intramedullary canal. Clin Orthop. 321: 111-116.

[17] Eastman, J., Tseng, S., Lo, E., et al. (2010). Retropateller technique for intramedullary nailing of proximal tibia fractures: A cadaveric assessment. J. Orthop Trauma. 24: 672- 676.

[18] Trueta, J. (1974). Blood supply and the rate of healing of tibial fractures. Clin Orthop Relat Res. 105: 11-26.

[19] Kirschner, M., Menck, J., Hennerbichlera et al. (1998). Importance OF arterial blood supply to the femur and tibia for transplantation of vascularized femoral diaphyses and knee joints. World J. Surg. 22: 845-851.

[20] Resnick, D., Newell, J., Guerra Jr, J., et al. (1978). Proximal tibiofibularjoimt: Anatomic, pathologic and radiologic correlation. Am J. Radiol. 131 (1): 133-138

[21] Kelly, P. (1968). Anatomy, physiology, and pathology of the blood supply of bones. Bone Joint Surg Am. 50: 766-783.

[22] Rhinelander, F. (1987) The vascular response of bone to internal fixation. In: Browner, B., Edwards, C. (eds.) The Science and Practice of Intramedullary Nailing, Lea \& Febiger, Philadelphia, pp. 25-29. 
[23] Strachan, R., McCarthy, I. Fleming, R., et al. (1990). The role of tibial nutrient artery. Microsphere estimation of blood flow in the osteotomized canine tibia. J. Bone Joint Surg Br. 72: 391-397.
[24] Rhinelander, F., Wilson, J. (1982). Blood supply to developing, mature and healing bone. In: Sumner-Smith, G: Bone in Clinical Orthopaedics: A Study of Comparative Osteology, Ch. 2., WB Saunders, Philadelphia, pp. $212-233$ 